\title{
Construcción de la Escala de Factores Personales de Resiliencia (FPR-1) en mujeres víctimas del desplazamiento forzado en Colombia*
}

\author{
Construction of Factores Personales de Resiliencia Scale \\ (FPR-1) in Victim's Woman of Forced Displacement in Colombia
}

Recibido: marzo 22 de 2013 | Revisado: enero 31 de 2014 | Aceptado: enero 31 de 2014

\author{
José JUAN AMAR AMAR ** \\ LEIDER MigUel UTRIA UTRIA *** \\ RAIMUNDO ABELLO LLANOS **** \\ MARINA BEGOÑa MARTíNEZ GONZÁLEZ ****** \\ Universidad del Norte, Colombia \\ Fernando Alexis CRESPo ROMERO ******* \\ Universidad Bernardo O'Higgins, Chile
}

doi:10.11144/Javeriana.UPSY13-3.cefp

Para citar este artículo: Amar, J. J., Utria, L. M., Abello, R., Martínez, M. B. \& Crespo, F. A. (2014). Construcción de la Escala de Factores Personales de Resiliencia (FPR-1) en mujeres víctimas del desplazamiento forzado en Colombia. Universitas Psychologica, 13(3), 853-864. http://dx.doi.org/10.11144/ Javeriana.UPSY13-3.cefp

* Artículo de investigación

** Decano de la División de Humanidades y Ciencias Sociales de la Universidad del Norte. Correo electrónico: jamar@uninorte.edu.co

*** Correo electrónico: leideru@uninorte.edu.co

****** Director de la Dirección de Investigación, Desarrollo e Innovación de la Universidad del Norte. Correo electrónico: rabello@uninorte.edu.co

******* Correo electrónico: martinezb@uninorte.edu.co

******** Dirección de Investigación. Correo electrónico: facrespo@gmail.com

\section{RES U MEN}

La escala FPR-1 se desarrolló con el fin de analizar los factores personales de resiliencia en un grupo de 113 mujeres desplazadas por la violencia en Colombia. Para esto, se llevó a cabo un estudio cuantitativo, explicativo, de corte psicométrico. El análisis factorial de la Escala señala la presencia de 13 factores latentes en torno a los cuales se organiza la información. Se encontró que las mujeres más resilientes son las adultas intermedias, seguidas de las mujeres mayores, mientras que las mujeres jóvenes tienden a la inconsistencia. Se destaca que la presencia de actitudes resilientes es explicada especialmente en relación con el altruismo y la apertura a los demás. Palabras clave

resiliencia; desplazamiento forzado; enfoque de género

\section{A B S T R A C T}

The PRF-1 scale was developed to analyze the personal factors of resilience in a group of 113 women displaced by violence in Colombia. For this we conducted a psychometric cutting, quantitative and explanatory study. Factor analysis of the scale indicates the presence of 13 latent factors around which the information is organized. We found out that women are more resilient at intermediate and older age, while young women are inconsistent. It's note that the presence of resilient attitudes is explained especially in relation to altruism and openness to others.

Keywords

resilience; forced displacement; human development 
José Juan Amar Amar, Leider Miguel Utria Utria, Raimundo Abello Llanos, marina Begoña Martínez González, Fernando Alexis Crespo Romero

El desplazamiento forzado es un fenómeno producto de la violencia sociopolítica que ha afectado durante muchos años a la población colombiana, convirtiéndose en una de las principales problemáticas de orden social en el país. Datos del Alto Comisionado de las Naciones Unidas para los Refugiados ([ACNUR]; 2009) y el VII informe de la Comisión de Seguimiento a la Política Pública sobre el Desplazamiento Forzado (2008), indican que existen más de 3.2 millones de desplazados, de los cuales cerca del $83 \%$ son mujeres y niños. Se señala también una alta tasa de hogares con jefatura femenina ( $43.4 \%$ de los registrados oficialmente).

Sobre el desplazamiento forzado se ha estudiado ampliamente el sufrimiento y la patología, pues esta situación se considera altamente traumática por los procesos de duelo y desarraigo de quienes la padecen, más no se ha profundizado en las potencialidades y recursos con los que cuentan estas personas, para afrontarla y superarla. Esto se debe en gran medida a que durante largo tiempo la psicología ha respondido a un modelo de salud mental entendida como ausencia de enfermedad y, por tanto, ha dirigido sus esfuerzos a la desaparición de los síntomas patológicos (Díaz, Blanco, Sutil \& Schweiger, 2007). Sin embargo, la mirada sobre salud, vista de forma integral, comienza a cambiar, reconociendo la coexistencia de elementos nocivos con elementos protectores de la salud en el mismo sujeto. El constructo teórico desde el cual se han estudiado los factores de afrontamiento que poseen los seres humanos para superar situaciones adversas se conoce como resiliencia, concepto tomado de la física para expresar la resistencia de un material para volver a su estado original, tras soportar altas presiones.

Existen estudios antecedentes sobre la resiliencia en personas desplazadas (Domínguez \& Godín, 2007). No obstante, pese a que las condiciones del género femenino en situación de desplazamiento son alarmantes, no se ha hecho un claro énfasis investigativo al respecto. El propósito de la investigación, fue desarrollar una escala con el fin de identificar los factores personales de resiliencia de mujeres en situación de desplazamiento, con base en el modelo teórico de Amar, Kotliarenco y
Abello (2004) que explora las dimensiones de autovaloración, autorregulación, habilidades sociales y competencias personales.

\section{Antecedentes y definición de la resiliencia}

A mediados del siglo pasado, las Ciencias Humanas comenzaron a utilizar el término resiliencia, para referirse a la capacidad de las personas para sobreponerse a situaciones adversas y sacar provecho de ellas. Las Ciencias Sociales adoptaron el término para caracterizar a las personas que nacen y viven en condiciones de alto riesgo, pero se desarrollan sanos y socialmente exitosos (Munist et al., 1998; Rutter, 1979). En este sentido la resiliencia, implica dos componentes: resistencia frente a la destrucción (capacidad de proteger la integridad ante las presiones deformantes) y capacidad para construir conductas vitales positivas (Cabrejos, 2005).

Las primeras investigaciones que exploraron esta característica no utilizaron el término resiliencia, sino que se refirieron a esta cualidad describiendo a dichos niños como invulnerables o invencibles, en el sentido de que eran resistentes al estrés (Masten, 2001). Aparece entonces otro concepto asociado a la resiliencia, el de invulnerabilidad, que puede definirse como la 'imposibilidad de ser vulnerado o herido'. Sin embargo, este concepto perdió su vigencia, por las siguientes razones (Rutter, 1993): (a) la invulnerabilidad implica una resistencia total al daño; (b) sugiere que se aplica a todas las circunstancias de riesgo; (c) implica una característica intrínseca del sujeto y (d) sugiere una característica estable en el tiempo.

Hoy, se entiende por resiliencia la 'capacidad para enfrentar, sobreponerse y ser fortalecido por experiencias de adversidad' (Grotberg, 2004). Por tanto, según el autor, se puede decir que se refiere a la capacidad de la persona para mantener un funcionamiento efectivo frente a las adversidades del entorno o para recuperarlo en otras condiciones. La resiliencia también se ha definido como la 'capacidad de una persona o grupo para proyectarse en el futuro pese a los acontecimientos desestabilizadores de la vida' (Kotliarenco, Cáceres \& Fontecilla, 
1997; Palomar \& Gómez, 2010; Suárez, 1996; Vera, Carbelo \& Vecina, 2006). Por su parte, Vanistendael (1997) plantea que la resiliencia requiere de la presencia de cinco dimensiones: 1) Existencia de redes sociales informales, 2) Sentido de vida, trascendencia, 3) Autoestima positiva, 4) Presencia de aptitudes y destrezas y 5) Sentido del humor.

Las conclusiones hoy permiten establecer que la resiliencia no es una característica absoluta, así como tampoco se adquiere para siempre. Más bien es el resultado de un proceso dinámico, con variaciones en función de la naturaleza del trauma, el contexto y la etapa de la vida en que ocurre, que además puede manifestarse de variadas formas según la cultura (Vera et al., 2006). Entre las variables relacionadas con la resiliencia que se han estudiado, se encuentran: características de personalidad y del entorno como la seguridad en sí mismo, el apoyo social, el propósito en la vida, la creencia en que se puede influir en lo que sucede alrededor y aprender de las experiencias positivas y negativas de la vida. También se ha encontrado que las personas resilientes conciben y afrontan la vida con más optimismo y energía, son personas abiertas a nuevas experiencias y con un alto nivel de emocionalidad positiva (Fredrickson \& Tugade, 2003).

Es importante destacar que la resiliencia se sustenta en la interacción entre la persona y el entorno, de manera que ni procede exclusivamente del entorno ni es algo puramente innato. Este proceso continuo en que se desarrollan persona y entorno hace que la resiliencia nunca sea absoluta ni terminantemente estable (Munist et al., 1998). La resiliencia permite una nueva epistemología del desarrollo humano, que atiende a las particularidades del contexto y llama a la responsabilidad multidisciplinaria de promoción de la calidad de vida (López, 2010).

\section{Medición de la resiliencia}

La resiliencia es un tema estudiado en la actualidad por la importancia que tiene para la promoción del desarrollo (Salgado, 2005). En las dos últimas décadas, se ha visto un aumento considerable en las investigaciones en este campo (Windle, Bennett, \&
Noyes, 2011). Hoy día, la resiliencia recibe atención especial de agentes políticos, como resultado de su potencial influencia en la salud, el bienestar y la calidad de vida y el cómo las personas responden a los distintos cambios a lo largo de su desarrollo (Friedli, 2009). En los estudios sobre la resiliencia, se identifican distintos enfoques para su medición, lo que ha dado lugar a incoherencias con respecto a la naturaleza de los factores de riesgo, los procesos de protección y las estimaciones de prevalencia de la resiliencia (Luthar, Cicchetti \& Becker, 2000). La medición se complica aún más debido a que se pueden anticipar tres escenarios de manifestación de la resiliencia: "que los individuos en riesgo muestren una mejor evolución a la esperada, que una adaptación positiva se mantenga a pesar de experiencias estresantes, o que se llegue a una buena recuperación después de un trauma" (Monroy \& Palacios, 2011, p. 238). En el rastreo de instrumentos de medición, se encuentra una sucinta documentación del tema. Entre estos instrumentos, existen estudios que han abordado la resiliencia como resistencia psicológica ante una situación difícil, pero caracterizándola como rasgo de personalidad: The Dispositional Resilience Scale, desarrollada por Bartone, Ursano, Wright y Ingraham (1989). Otras posturas han buscado factores asociados a la capacidad de afrontamiento del estrés: The Connor-Davidson Resilience Scale (CD-RISC) desarrollada por Connor y Davidson (1999), así como factores protectores intrapersonales e interpersonales que favorecen la resiliencia: The Resilience Scale for Adults - RSA, propuesta por Friborg, Hjemdal, Rosenvinge y Martinussen (2003); y la Resilience Scale for Adolescents (READ) construida por Hjemdal, Friborg, Stiles, Martinussen y Rosenvinge (2006). También se han explorado las actitudes resilientes en jóvenes, con el instrumento The Resiliency Attitudes and Skills Profile, desarrollado por Hurtes y Lawrence (2001). De igual forma, se encontraron algunas escalas como The Resilience Scale (RS) propuesta por Wagnild y Young (1993), desarrollada en Australia, la cual se enfoca en identificar el grado de resiliencia individual en tres sentidos: competencia personal, aceptación de uno mismo y de la vida. En 
José Juan Amar Amar, Leider Miguel Utria Utria, Raimundo Abello Llanos, marina Begoña Martínez González, Fernando Alexis Crespo Romero

el Reino Unido, también se encuentra una escala denominada Psychological Resilience, desarrollada por Windle, Markland y Woods (2008), que evalúa la capacidad de recuperación psicológica asociada a factores que actúan como protección contra los riesgos y las adversidades. En el caso latinoamericano, se encontró una escala de medición de la resiliencia con mexicanos (RESI-M), desarrollada por Lever y Gómez (2010) quienes se basaron para su construcción en las escalas CD-RISC y la RSA, mencionadas anteriormente. En Perú, se encontró el Inventario de Factores Personales de Resiliencia, desarrollado por Salgado (2005) que evalúa los factores de autoestima, empatía, autonomía, humor y creatividad, en niños.

\section{Modelo teórico de la resiliencia}

La línea de partida para la investigación citada en este artículo es el modelo analítico comprensivo de la resiliencia, planteado por Amar, Kotliarenco y Abello (2004), desarrollado con niños víctimas activas y pasivas de violencia intrafamiliar, a partir de una investigación cualitativa basada en la metodología de la teoría fundamentada. Los resultados de dicha investigación coincidieron con investigaciones en este campo: los niños resilientes presentan características y factores que los protegen de la adversidad y les permiten un desarrollo psicosocial positivo en su autovaloración, autorregulación, competencias individuales y habilidades sociales.

\section{Método}

\section{Diseño}

La investigación desarrollada es cuantitativa, explicativa, con un diseño no experimental, transversal y de corte psicométrico.

\section{Participantes}

La muestra de carácter intencional, fue conformada por 113 mujeres en situación de desplazamiento forzado por la violencia sociopolítica, radicadas en la ciudad de Barranquilla.

\section{Instrumento}

La escala FPR-1 se desarrolló con el fin de evaluar la resiliencia en mujeres desplazadas por la violencia en Colombia. La línea teórica de partida fue el modelo analítico comprensivo de la resiliencia, planteado por Amar, Kotliarenco y Abello, (2004). Los factores de la resiliencia se definieron operacionalmente a partir de las siguientes categorías:

- Autovaloración: percepción positiva o negativa que se tiene de sí mismo.

- Autorregulación: manera como se aceptan las responsabilidades y las normas.

- Competencias: estimación de las capacidades para interactuar con los demás y con el mundo.

- Habilidades sociales: actitudes en el plano interpersonal.

Se construyó una escala tipo Likert, con tres opciones de respuesta: en desacuerdo (1), ni de acuerdo ni en desacuerdo (2) y de acuerdo (3). Puntajes cercanos a 3, darían cuenta de un mayor nivel de resiliencia y cercanos a 2 una actitud inconsistente o disonante.

\section{Procedimiento}

Después de la revisión del estado del arte, se construyó una escala inicial de 66 ítems que fue sometida a un proceso de validación de expertos y un pilotaje con la población del estudio. Se tramitaron los permisos respectivos para acceder a un grupo de mujeres en situación de desplazamiento que reciben atención en las instalaciones de una ONG operadora del Estado en la ciudad de Barranquilla. El estudio fue presentado a los participantes como una investigación sobre aquellas características que les han permitido superar las dificultades en la vida. Se les explicó el consentimiento informado y se les entregó la escala a quienes aceptaron participar. Algunas mujeres solicitaron ayuda para diligenciar el cuestionario, pues eran analfabetas. Tras la aplicación del instrumento, se procedió a la digitación de los datos y su posterior análisis, utilizando el programa IBM SPSS Statistics 20. 


\section{Resultados}

\section{Validez de constructo}

La escala inicial estuvo conformada por 66 ítems a los cuales se aplicó un proceso de validación de contenido por jueces expertos. Su evaluación permitió decantar la escala hasta quedar conformada por 33 ítems, como sigue: cinco ítems para autovaloración, siete para la autorregulación, once para competencias personales y diez para habilidades sociales. Se plantearon ítems inversos con el fin de evitar la aquiescencia al momento de responder la prueba.

\section{Análisis factorial}

Mediante un análisis factorial basado en componentes principales, se buscó probar si la información de las respuestas se refería a las cuatro categorías del modelo teórico inicial. Sin embargo, tal como se evidencia en la Tabla 1, el análisis inicial de reducción de variables con componentes principales, reveló que existen 13 factores latentes, que completan más del $80 \%$ de la información sobre la varianza, de manera que los ítems no están concentrados en torno de los primeros factores.

Las correlaciones en los primeros factores resultaron pequeñas, y aparecen diversas variables explicativas en los primeros de ellos. Desde el sexto factor, ya no fue posible identificar con claridad cuál ítem se asocia con fuerza a cuál factor. Para corregir este problema, se aplicó la solución rotada con el criterio VARIMAX. Con ello se obtuvieron valores que presentan mayor fuerza de asociación respecto de las variables iniciales que se aprecian en la Tabla 2. Esto permitió encontrar la agrupación de ítems entorno a 13 factores como sigue:

\section{Consistencia interna}

El coeficiente $\alpha$ de la escala FPR-1 señala, en general, una consistencia interna aceptable, con un indicador de 0.58. El análisis de la confiabilidad para cada uno de los factores de la escala (Tabla 2) mejora para el factor de Autovaloración con Orientación Social con un alfa de Cronbach de 0.73 y para el factor de Autoplanificación, con un índice de 0.74. Sin embargo, empeora para los factores de Percepción de Autoeficacia con un índice de 0.39, Espontaneidad Social sin Control de las Emociones con un índice de 0.51, Asertividad y Afrontamiento con un índice de 0.43, Cumplimiento de Rutinas y Normas con un índice de 0.4 y Adaptación a las Circunstancias con un índice de confiabilidad de 0.45. Se encuentra que en algunos de estos casos la confiabilidad mejora si se elimina alguno de

TABLA 1

Eigenvalues (valores propios), porcentajes de varianzas y porcentajes acumulados de los factores del cuestionario

\begin{tabular}{cccc}
\hline Factor & Valores propios & \% de varianza & \% acumulativo \\
\hline 1 & 5.6 & 16.9 & 16.9 \\
2 & 2.9 & 8.9 & 25.7 \\
3 & 2.8 & 8.6 & 34.3 \\
4 & 2.4 & 7.4 & 41.7 \\
5 & 2 & 6.1 & 47.8 \\
6 & 1.9 & 5.8 & 53.6 \\
7 & 1.7 & 5.2 & 58.7 \\
8 & 1.5 & 4.5 & 63.2 \\
9 & 1.4 & 4.1 & 67.3 \\
10 & 1.2 & 3.6 & 70.9 \\
11 & 1.1 & 3.4 & 74.3 \\
12 & 1 & 3.2 & 77.4 \\
13 & 1 & 3 & 80.4 \\
\hline
\end{tabular}

Fuente: elaboración propia 
TABLA 2

Análisis de confiabilidad y cargas factoriales por rotación ortogonal VARIMAX

\begin{tabular}{|c|c|c|c|c|}
\hline Factor & $\begin{array}{c}\alpha \text { de } \\
\text { Cronbach }\end{array}$ & $\begin{array}{c}\alpha \text { de } \\
\text { Cronbach si } \\
\text { se elimina } \\
\text { elemento }\end{array}$ & Sig. & Ítem \\
\hline \multirow{5}{*}{$\begin{array}{l}\text { Autovaloración con } \\
\text { Orientación Social }\end{array}$} & \multirow{5}{*}{0.73} & 0.664 & 0.703 & Soy una persona que se quiere a sí misma. \\
\hline & & 0.708 & 0.873 & Puedo apoyar a otros que tienen problemas. \\
\hline & & 0.681 & 0.907 & Soy capaz de ayudar a otras personas. \\
\hline & & 0.693 & 0.499 & Puedo hablar de mis emociones con otros. \\
\hline & & 0.675 & 0.54 & Disfruto de estar con otras personas. \\
\hline \multirow{3}{*}{ Autoplanificación } & \multirow{3}{*}{0.74} & 0.617 & 0.799 & Soy responsable con las decisiones que tomo. \\
\hline & & 0.739 & 0.756 & Planifico y organizo mis actividades. \\
\hline & & 0.645 & 0.84 & Sé muy bien lo que quiero para mi vida. \\
\hline \multirow{3}{*}{ Percepción de Autoeficacia } & \multirow{3}{*}{0.39} & 0.094 & 0.692 & Soy un modelo positivo para otros. \\
\hline & & 0.671 & 0.581 & No me siento satisfecho con la vida que llevo. \\
\hline & & & 0.865 & He tenido logros en mi vida. \\
\hline \multirow{3}{*}{$\begin{array}{l}\text { Espontaneidad Social sin } \\
\text { Control de las Emociones }\end{array}$} & \multirow{3}{*}{0.51} & 0.803 & 0.486 & Es difícil controlarme cuando me enojo. \\
\hline & & 0.359 & 0.867 & Puedo comunicarme bien con otras personas. \\
\hline & & & 0.809 & Puedo comenzar una conversación. \\
\hline \multirow[b]{2}{*}{ Asertividad y Afrontamiento } & \multirow[b]{2}{*}{0.43} & 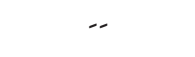 & 0.751 & Soy capaz de dar mi opinión en situaciones difíciles. \\
\hline & & - & 0.749 & $\begin{array}{l}\text { Enfrentar situaciones difíciles me ayuda a afrontar } \\
\text { mejor la vida. }\end{array}$ \\
\hline Vinculación Familiar & $\begin{array}{l}\text { El valor es } \\
\text { cual viola } \\
\text { del modelo }\end{array}$ & $\begin{array}{l}\text { s negativo lo } \\
\text { los supuestos } \\
\text { o de fiabilidad }\end{array}$ & $\begin{array}{l}0.815 \\
-0.676\end{array}$ & $\begin{array}{l}\text { Mantengo una buena relación con mi familia. } \\
\text { Se me dificulta expresar cariño a mis familiares. }\end{array}$ \\
\hline \multirow{2}{*}{$\begin{array}{l}\text { Cumplimiento de Rutinas y } \\
\text { Normas }\end{array}$} & \multirow{2}{*}{0.4} & -. & 0.908 & No cumplo los planes que me propongo. \\
\hline & & - & 0.504 & Me cuesta trabajo seguir las normas de mi comunidad. \\
\hline \multirow{3}{*}{$\begin{array}{l}\text { Adaptación a las } \\
\text { Circunstancias }\end{array}$} & \multirow{3}{*}{0.45} & \multirow{3}{*}{$\begin{array}{l}0.34 \\
0.197 \\
0.496\end{array}$} & 0.483 & No me siento satisfecho con la vida que llevo. \\
\hline & & & 0.884 & Me cuesta adaptarme a las situaciones nuevas. \\
\hline & & & 0.45 & Es difícil controlarme cuando me enojo. \\
\hline Apertura Social & \multicolumn{2}{|c|}{$\begin{array}{l}\text { No hay suficientes } \\
\text { elementos }\end{array}$} & 0.904 & Soy capaz de hacer nuevas amistades. \\
\hline Autodeterminación & \multicolumn{2}{|c|}{$\begin{array}{l}\text { No hay suficientes } \\
\text { elementos }\end{array}$} & 0.878 & $\begin{array}{l}\text { Dependo mucho de otras personas para tomar mis } \\
\text { decisiones. }\end{array}$ \\
\hline Sentido del Humor & \multicolumn{2}{|c|}{$\begin{array}{l}\text { No hay suficientes } \\
\text { elementos }\end{array}$} & 0.893 & Es fácil para mí hacer reír a otras personas. \\
\hline Visión del Futuro & \multicolumn{2}{|c|}{$\begin{array}{l}\text { No hay suficientes } \\
\text { elementos }\end{array}$} & 0.859 & Soy pesimista respecto del futuro. \\
\hline Gestión Social & \multicolumn{2}{|c|}{$\begin{array}{l}\text { No hay suficientes } \\
\text { elementos }\end{array}$} & 0.888 & $\begin{array}{l}\text { No logro hacer amigos que me apoyen cuando tengo } \\
\text { problemas. }\end{array}$ \\
\hline
\end{tabular}

Fuente: elaboración propia

los ítems, generalmente los que tienen una carga negativa.

\section{Análisis descriptivo de la Escala FPR-1}

Un total de 113 mujeres respondió a la aplicación de la escala, pero solo 48 diligenciaron todos los ítems.
El promedio de edad encontrado fue de 39.43 años $(D E=12.74)$, con un rango entre los 18 y 74 años.

En la Tabla 3, se ven los efectos por variable separada, donde se observa que aquellas que permiten diferenciar los grupos son la edad y el ítem "Soy capaz de ayudar a otras personas", dado que tienen valor de significancia menor que 0.05 . 
TABLA 3

Efectos de variable sobre el clúster

\begin{tabular}{|c|c|c|}
\hline Variable & $F(2,42)$ & $p$ \\
\hline EDAD & 116.158 & 0 \\
\hline Soy una persona que se quiere a sí misma. & 0.57 & 0.57 \\
\hline Soy un modelo positivo para otros. & 2.076 & 0.138 \\
\hline No me siento satisfecho con la vida que llevo. & 0.515 & 0.601 \\
\hline He tenido logros en mi vida. & 1.491 & 0.237 \\
\hline No sé cuáles son mis habilidades. & 0.645 & 0.53 \\
\hline Tengo el control de mi vida. & 0.382 & 0.685 \\
\hline Soy pesimista respecto del futuro. & 0.137 & 0.873 \\
\hline Soy responsable con las decisiones que tomo. & 1.598 & 0.214 \\
\hline Me cuesta adaptarme a las situaciones nuevas. & 0.693 & 0.506 \\
\hline Es difícil controlarme cuando me enojo. & 0.322 & 0.726 \\
\hline No cumplo los planes que me propongo. & 1.192 & 0.314 \\
\hline Me cuesta trabajo seguir las normas de mi comunidad. & 0.116 & 0.891 \\
\hline Planifico y organizo mis actividades. & 0.055 & 0.946 \\
\hline Dependo mucho de otras personas para tomar mis decisiones. & 1.902 & 0.162 \\
\hline Soy capaz de dar mi opinión en situaciones difíciles. & 1.506 & 0.234 \\
\hline No busco ayuda cuando la necesito. & 1.75 & 0.186 \\
\hline Puedo apoyar a otros que tienen problemas. & 1.262 & 0.294 \\
\hline Puedo comunicarme bien con otras personas. & 1.774 & 0.182 \\
\hline Sé muy bien lo que quiero para mi vida. & 0.067 & 0.936 \\
\hline Enfrentar situaciones difíciles me ayuda a afrontar mejor la vida. & 0.592 & 0.558 \\
\hline Puedo hablar sobre las cosas que me asustan o me inquietan. & 0.657 & 0.524 \\
\hline Puedo comenzar una conversación. & 0.101 & 0.904 \\
\hline No puedo resolver mis problemas personales. & 0.444 & 0.645 \\
\hline Soy capaz de hacer nuevas amistades. & 1.341 & 0.272 \\
\hline Mantengo una buena relación con mi familia. & 1.898 & 0.162 \\
\hline No logro hacer amigos que me apoyen cuando tengo problemas. & 0.613 & 0.547 \\
\hline Es fácil para mí hacer reír a otras personas. & 0.124 & 0.883 \\
\hline Soy capaz de ayudar a otras personas. & 3.107 & 0.055 \\
\hline Puedo hablar de mis emociones con otros. & 1.362 & 0.267 \\
\hline Disfruto de estar con otras personas. & 0.809 & 0.452 \\
\hline Se me dificulta expresar cariño a mis familiares. & 2.181 & 0.126 \\
\hline No disfruto pasar tiempo con mi familia. & 0.515 & 0.601 \\
\hline Confío en otras personas. & 1.213 & 0.308 \\
\hline
\end{tabular}

Fuente: elaboración propia

De acuerdo con esto, las actitudes resilientes estarían fuertemente relacionadas con una tendencia a la ayuda de los demás, una apertura hacia el otro. Se encuentra que las mujeres en adultez intermedia (edad promedio 38 años) y las mujeres mayores (edad promedio 57 años) son las que presentan un mayor índice en la variable explicativa $(\mathrm{M}=3 ; \mathrm{DE}=0 \mathrm{y} \mathrm{M}$ $=2.94, \mathrm{DE}=0.25$; respectivamente), en contraste con las mujeres jóvenes (edad promedio 26 años) que presentan el puntaje más bajo, si bien conservan una tendencia positiva $(\mathrm{M}=2.62 ; \mathrm{DE}=0.77)$.

En la Tabla 4, se puede analizar el comportamiento de los clústeres en relación con cada uno de los factores de la escala.

Se encuentra que para el factor de Autovaloración con Orientación Social, el grupo de mujeres 
José Juan Amar Amar, Leider Miguel Utria Utria, Raimundo Abello Llanos, marina Begoña Martínez González, Fernando Alexis Crespo Romero

TABLA 4

Medias obtenidas por los clústeres en cada factor

\begin{tabular}{ccccccccc}
\hline & \multicolumn{9}{c}{ Cluster } \\
\cline { 2 - 9 } & \multicolumn{1}{c}{ Mujeres mayores } & \multicolumn{2}{c}{ Mujeres jóvenes } & \multicolumn{2}{c}{ Mujeres intermedias } & \multicolumn{2}{c}{ Total } \\
\hline Factor & $\mathrm{M}$ & $\mathrm{DE}$ & $\mathrm{M}$ & $\mathrm{DE}$ & $\mathrm{M}$ & $\mathrm{DE}$ & $\mathrm{M}$ & $\mathrm{DE}$ \\
\hline 1 & 2.78 & 0.56 & 2.63 & 0.74 & 2.93 & 0.26 & 2.79 & 0.57 \\
2 & 2.88 & 0.38 & 2.82 & 0.57 & 2.9 & 0.35 & 2.87 & 0.45 \\
3 & 2.38 & 0.93 & 2.33 & 0.87 & 2.73 & 0.62 & 2.49 & 0.83 \\
4 & 2.56 & 0.57 & 2.69 & 0.49 & 2.71 & 0.45 & 2.65 & 0.52 \\
5 & 2.59 & 0.71 & 2.69 & 0.67 & 2.56 & 0.76 & 2.61 & 0.73 \\
6 & 2.16 & 0.58 & 2.23 & 0.89 & 2.06 & 0.69 & 2.14 & 0.74 \\
7 & 1.63 & 0.92 & 1.73 & 0.89 & 1.91 & 0.93 & 1.76 & 0.91 \\
8 & 2.31 & 0.88 & 2.21 & 0.95 & 2.42 & 0.85 & 2.32 & 0.88 \\
9 & 2.94 & 0.25 & 3 & 0 & 2.75 & 0.68 & 2.89 & 0.44 \\
10 & 1.75 & 0.93 & 1.15 & 0.55 & 1.63 & 0.96 & 1.53 & 0.87 \\
11 & 2.63 & 0.62 & 2.69 & 0.63 & 2.56 & 0.81 & 2.62 & 0.68 \\
12 & 2.19 & 0.98 & 2 & 1 & 2.06 & 1 & 2.09 & 0.97 \\
13 & 1.69 & 0.95 & 1.92 & 0.95 & 2.06 & 1 & 1.89 & 0.96 \\
\hline
\end{tabular}

Fuente: elaboración propia

en adultez intermedia tiene el mejor puntaje, en contraste con las mujeres jóvenes. De igual forma ocurre con el factor de Autoplanificación, donde las mujeres adultas intermedias obtienen mejor puntaje que las más jóvenes y en el factor de Percepción de Autoeficacia, donde las diferencias son más significativas entre el grupo de las mujeres intermedias y el de las jóvenes y mayores, que tienden a la inconsistencia. La Espontaneidad Social sin Control de las Emociones está más presente en las mujeres desplazadas en adultez intermedia.

Se encontró que la Gestión Social tuvo puntajes inconsistentes para las mujeres intermedias y las jóvenes, mientras que las mujeres mayores obtienen un puntaje bajo dando cuenta de que a mayor edad, más escasa presencia de esta variable.

El Cumplimiento de Rutinas y Normas presentó una tendencia a la inconsistencia en las mujeres intermedias y las jóvenes, mientras que las mujeres mayores obtienen un puntaje bajo. Dado que estos indicadores en la escala son inversos, los puntajes señalan que las mujeres mayores tienen menos dificultad para ajustarse a las normas del contexto y a las rutinas.

En lo referente a factores como la Ssertividad y Capacidad de Afrontamiento y el Sentido del
Humor no se encuentran diferencias significativas entre los grupos y la tendencia es a una buena puntuación para estos factores.

La Apertura Social fue el factor con puntaje más alto en todos los grupos, especialmente en las mujeres jóvenes que son seguidas de las mujeres mayores y finalmente las intermedias.

La Vinculación Familiar, la Adaptación a las Circunstancias y la Visión del Futuro son los factores más inconsistentes para todos los grupos. En el caso de la Autodeterminación, los puntajes aunque más bajos también tienden a la inconsistencia en las mujeres mayores e intermedias, mientras que en las jóvenes escasea la presencia de esta característica.

\section{Discusión}

El presente estudio tuvo como objetivo conocer las actitudes resilientes de mujeres en situación de desplazamiento forzado por la violencia, para lo cual se desarrolló la escala de medición de la resiliencia FPR-1. La forma como se reorganizaron los ítems en la escala, si bien no corresponde en estricto sentido al modelo teórico de cuatro categorías planteado inicialmente, se puede pensar en los nuevos factores como subcomponentes de estas de acuerdo 
a su definición conceptual. Para esto, se sugieren posteriores revisiones a la escala.

Es importante mencionar que algunas dificultades en el proceso de validación se debieron a que la muestra de 135 mujeres no respondió la totalidad de las preguntas, tal como estaban en libertad de hacerlo, pues se expresa en el consentimiento informado. Se parte del reconocimiento de que la situación de desplazamiento ha provocado daños y pérdidas que aún duelen en las víctimas y que la resiliencia no es un estado absoluto, sino una cualidad que se manifiesta en diversos aspectos de la vida de la persona, sin negar dificultades para el funcionamiento en algunos otros. Esto puede llevarlas a optar por no responder preguntas que les resultan incómodas. Sin embargo, dado que se pretendía dar cuenta de los aspectos con funcionamiento positivo, los hallazgos resultan un valioso conocimiento sobre las características de las mujeres en situación de desplazamiento.

En términos generales, la escala permitió identificar los factores asociados a la resiliencia en mujeres desplazadas por la violencia, muchos de ellos coincidentes con las construcciones teóricas desarrolladas sobre el concepto, pero señalando nuevas comprensiones basadas en la manera como dichos factores se expresan en ellas.

Entre los elementos coincidentes con el estado del arte, se encuentra que las mujeres en situación de desplazamiento, desarrollan una adaptación y capacidad de afrontamiento a las nuevas circunstancias. Son muchos los estudios que han planteado que la resiliencia incluye la aceptación de los problemas como parte de la vida y la posibilidad de adaptarse a las pérdidas gracias a la valoración de lo aprendido (Cabrejos, 2005; Grotberg, 2004; Palomar \& Gómez, 2010; Rutter, 1979; Vera et al., 2006).

En el estudio, también aparece el sentido del humor como un vehículo para la relación con los demás, lo que, de acuerdo con anteriores investigaciones (Menoni \& Klasse, 2007), permite encontrar alivio, incluso en situaciones muy adversas.

La visión de futuro y la autoplanificación para el logro de objetivos vitales, también son factores de resiliencia que aparecen en las mujeres despla- zadas por la violencia. Estas se han estudiado en relación con el bienestar y la resiliencia, debido a que influyen en los comportamientos presentes y se vinculan con la toma de decisiones, y además, pueden modificar la manera de adaptarse a las experiencias nefastas, reduciendo su percepción como amenazas (Omar, 2009).

En lo referente a la vinculación familiar, los resultados señalan la capacidad de las mujeres participantes para llevar las relaciones al interior de la familia, de manera que quienes mantienen una buena relación son aquellas que realizan más demostraciones de afecto. Diversos estudios indican que contar con relaciones que ofrezcan cuidado y apoyo dentro y fuera de la familia, generan estímulo y reafirmación de la resiliencia en la persona (Domínguez, 2005).

En cuanto a los hallazgos que sugieren una reinterpretación del concepto, se encuentra, por ejemplo, lo identificado sobre la percepción de autoeficacia. Benight y Bandura (2004) ya habían planteado que la valoración de las propias capacidades ayuda a la superación de situaciones traumáticas como desastres naturales, catástrofes tecnológicas, ataques terroristas, combates militares y asaltos sexuales criminales. Sin embargo, es necesario destacar que en la presente investigación, la autoeficacia está asociada a la insatisfacción con la vida. Esto resulta paradójico, debido a que la satisfacción con la vida se ha estudiado durante mucho tiempo como un indicador salugénico (Diener, 2000).

Ahora bien, en el contexto colombiano, estudios realizados con población desplazada han encontrado que estas personas mantienen altos niveles de satisfacción con la vida que, sin embargo, correlacionan positivamente con indicadores de fatalismo (Abello et al., 2009). Los investigadores concluyen que esto podría ser una manifestación de la aceptación pasiva de la realidad, asumida por las personas desplazadas. En la presente investigación, ocurre lo contrario. Al parecer, las mujeres desplazadas que se perciben más autoeficaces se sienten menos satisfechas con las condiciones de vida que llevan. Aunque la resiliencia se ha contemplado como un proceso de mejora después de la experiencia traumática (Vera et al., 2006), al parecer se manifiesta 
de forma concomitante con la situación de estrés para las personas desplazadas, que resulta permanente al tener que vivir bajo este estigma para poder acceder a los servicios del Estado, además de que sus derechos no han sido plenamente restablecidos. La insatisfacción con estas circunstancias, más que ser un indicador de patología, es un indicador de deseo de superación, que señalaría una actitud resiliente cuando la persona permanece en la situación traumática.

Otro elemento que se destaca es lo encontrado en relación al factor de autovaloración con orientación social. Este señala el reconocimiento de la valía propia en la relación con los demás, de manera que en las mujeres desplazadas resilientes, especialmente aquellas en edad intermedia, la propia valoración conduciría a una apertura hacia los demás y una disposición hacia el altruismo. Las respuestas de las mujeres desplazadas también permitieron identificar un factor asociado a la apertura social, entendida como la disposición a la búsqueda de amigos. Se ha encontrado que otro tipo de víctimas, en este caso niños víctimas de violencia intrafamiliar que presentan actitudes resilientes, se consideran felices porque pueden jugar con sus amigos, compartir experiencias agradables con ellos y experimentar la aceptación de estos (Amar et al., 2004). Al parecer, el desarrollo de la resiliencia requiere de la presencia de redes sociales informales, amigos con quienes participar de actividades y la capacidad de mantener buenas relaciones (Vanistendael, 1997).

Por otra parte, se ha encontrado una relación positiva entre la autoestima y el desarrollo de conductas prosociales, de manera que la persona que se valora a sí misma, muestra iniciativa para emprender acciones por otras personas. Así, el altruismo y la empatía se han estudiado como factores asociados a la resiliencia (Palomar \& Gómez, 2010; Vanistendael, 1997). En el contexto de la resiliencia, la empatía implica la posibilidad del sujeto para ponerse en el lugar del otro y reevaluar el daño que le han hecho (Kotliarenco et al., 1997), lo cual es importante para las víctimas de desplazamiento forzado y la manera como asumen a sus victimarios, con miras a procesos de paz y la reinserción social de estos actores a la vida civil.
De igual forma, resulta interesante lo encontrado en el factor de espontaneidad social sin control de las emociones el cual señala la capacidad de la persona para comunicarse y relacionarse con los demás, pero asociado a dificultades en el control de las emociones. Este factor agrupa variables que indicarían la presencia de una tendencia a relacionarse con el otro, pero que en las mujeres en situación de desplazamiento se caracteriza por la dificultad para el manejo de las emociones. Asimismo, surge el factor de incumplimiento de rutinas y normas como parte de las actitudes resilientes en las mujeres desplazadas participantes.

Según Vanistendael (1995), la resiliencia incluye la capacidad de la persona para enfrentar las dificultades de una forma socialmente aceptable y correcta. Palomar y Gómez (2010) han estudiado la capacidad de las personas para seguir reglas y actividades sistémicas en momentos difíciles como un indicador de resiliencia. En el caso de las personas en situación de desplazamiento, se identifica que a su llegada tienden a ubicarse en los cinturones de miseria de las ciudades receptoras, encontrándose con una serie de situaciones y hábitos sociales que podrían chocar con las tradiciones a las que están acostumbradas en la vida del campo. Esto podría justificar que las mujeres en situación de desplazamiento sientan que no logran seguir las normas sociales de las comunidades receptoras, especialmente las más jóvenes y las de edad intermedia.

En relación con esto, también se encuentra el factor de autodeterminación, el cual señala la percepción de la persona sobre su capacidad o, por el contrario, dependencia de terceros para la toma de decisiones. La competencia para evaluar los acontecimientos de manera que faciliten un afrontamiento eficaz están también implícitas en los procesos de protección a la adversidad y, en su defecto, en el mantenimiento de diversos trastornos psicológicos y en lo que algunos han denominado "vulnerabilidad psicológica" (Lemos, 2005). En este caso, las mujeres desplazadas con menos actitudes resilientes serían más vulnerables a la influencia social, más dependientes de terceros para la toma de sus decisiones. 
Estos hallazgos dan cuenta de la presencia y ausencia de estos factores de resiliencia en relación a los grupos de edad, de manera que los programas de intervención psicosocial ofrecidos en las entidades que atienden a esta población podrían enfocar sus actividades más específicamente de acuerdo al momento evolutivo, con énfasis en el desarrollo de habilidades sociales, la autorregulación emocional y la autodeterminación que estimulen una mejor adaptación social en la comunidad receptora.

\section{Referencias}

Abello, R., Amarís, M., Blanco, A., Madariaga, C., Manrique, K., Martínez, M., Turizo, Y., et al. (2007). Bienestar y trauma en personas adultas desplazadas por la violencia política. Universitas Psychologica, 8(2), 455-470.

Alto Comisionado de las Naciones Unidas para los Refugiados. (2009). Violencia de género y mujeres desplazadas. Recuperado de http://www.acnur.org/ t3/fileadmin/Documentos/RefugiadosAmericas/ Colombia/Violencia_de_genero_y_mujeres_desplazadas.pdf?view $=1$

Amar, J., Kotliarenco, M. A. \& Abello, R. (2004). Resiliencia en niños víctimas de violencia intrafamiliar. Ensayos en Desarrollo Humano, 5. Barranquilla: Ediciones Uninorte.

Bartone, P. T., Ursano, R. J., Wright, K. M. \& Ingraham, L. H. (1989). The impact of a military air disaster on the health of assistance workers: A prospective study. The Journal of Nervous and Mental Disease, 177(6), 317-328.

Benight, C. \& Bandura, A. (2004). Social cognitive theory of posttraumatic recovery: The role of perceived self-efficacy. Behaviour Research and Therapy, 42(10), 1129-1148.

Cabrejos, J. (2005). La promoción de la resilencia y el diseño de políticas sociales. Revista de la Facultad de Ciencias Económicas de la Universidad Nacional Mayor de San Marcos, 10(28), 47-70. Recuperado de http://sisbib.unmsm.edu.pe/bibvirtualdata/ publicaciones/economia/28/a04.pdf

Comisión de Seguimiento a la Política Pública sobre el Desplazamiento Forzado (2008). Séptimo In- forme De Verificación Sobre El Cumplimiento De Derechos De La Población En Situación De Desplazamiento. Recuperado de: http://www.acnur.org/t3/uploads/media/COI_2492.pdf?view $=1$

Connor, K. M. \& Davidson, J. R. T. (1999). Development of a new resilience scale: The Connor - Davidson Resilience Scale (CDRISC). Depression and Anxiety, 18(2), 76-82.

Díaz, D., Blanco, A., Sutil, L. \& Schweiger, I. (2007). Argumentos para una propuesta psicosocial del trauma: el bienestar, patrón de salud y de trastorno. En S. Yubero, E. Larrañaga, y A. Blanco (Coords.) (2007). Convivir con la violencia. Cuenca: Ediciones de la Universidad Castilla-La Mancha.

Diener, E. (2000). Subjective well-being. The science of happiness and a proposal for a national index. American Psychologist, 55(1), 34-43. http://dx.doi. org/10.1037/0003-066X.55.1.34

Domínguez, J. (2005). Resiliencia después del huracán Katrina y Rita. Recuperado de www.apa-helpcenter. org/articles/article.php?id=114

Domínguez, E. \& Godín, R. (2007). La resiliencia en familias desplazadas por la violencia sociopolítica ubicadas en Sincelejo. Psicología desde el Caribe, 19, 154-180.

Fredrickson, B. L. \& Tugade, M. M. (2003). What good are positive emotions in crises? A prospective study of resilience and emotions following the terrorist attacks on the USA on September 11th, 2001. Journal of Personality and Social Psychology, 84(2), 365 376. http://dx.doi.org/10.1037/0022-3514.84.2.365

Friborg, O., Hjemdal, O., Rosenvinge, J. H. \& Martinussen, M. (2001). A new rating scale for adult resilience: What are the central protective resources behind healthy adjustment? International Journal of Methods in Psychiatric Research, 12(2), 65-76.

Friedli, L. (2009). Mental health, resilience and inequalities: A report for WHO Europe and the Mental Health Foundation. London/Copenhagen: Mental Health Foundation/WHO Europe. Recuperado de http://www.euro.who.int/document/e92227.pdf

Grotberg, E. (2004). Nuevas tendencias en resiliencia [Introducción]. En A Melillo \& N. Suárez (Comps.), Resiliencia: descubriendo las propias fortalezas (pp. 19-30). Buenos Aires: Paidós. 
José Juan Amar Amar, Leider Miguel Utria Utria, Raimundo Abello llanos, marina Begoña Martínez González, Fernando Alexis Crespo Romero

Hjemdal, O., Friborg, O., Stiles, T. C., Martinussen, M. \& Rosenvinge, J. H. (2006). A new rating scale for adolescent resilience: Grasping the central protective resources behind healthy development. Measurement and Evaluation in Counseling and Development, 39(2), 84-96.

Hurtes, P. K. \& Lawrence, R. A. (2001). Measuring resiliency in youth: The resiliency attitudes and skills profile. Therapeutic Recreation Journal, 35(4), 333-348.

Kotliarenco, M. A., Cáceres, I. \& Montecilla, M. (1997). Estado de arte en resiliencia. Organización Panamericana de la Salud, Oficina Sanitaria Panamericana/ Organización Mundial de la Salud, Centro de Estudios de Atención al Niño y la Mujer. Recuperado de http://www.paho.org/Spanish/HPP/ HPF/ADOL/Resil6x9.pdf

Lemos, S. (2005). Variables cognitivas. En L. Ezpeleta (Ed.), Factores de riesgo en psicopatología del desarrollo (pp. 147-176). Barcelona: Masson.

López, V. (2010). Educación y resiliencia: alas de la transformación social. Actualidades Investigativas en Educación, 10(2), 1-14. Recuperado de http:// revista.inie.ucr.ac.cr/articulos/2-2010/archivos/ resiliencia.pdf

Luthar, S., Cicchetti, D. \& Becker, B. (2000). The construct of resilience: A critical evaluation and guidelines for future work. Child Development, 71(3), 543 562. http://dx.doi.org/10.1111/1467-8624.00164

Masten, A. S. (2001). Ordinary magic: Resilience processes in development. American Psychologist, 56(3), 227 - 238. http://dx.doi.org/10.1037/0003066X.56.3.227

Menoni, T. \& Klasse, E. (2007). Construyendo alternativas al dolor: reflexiones sobre la resiliencia en barrio Casabó, cerro de Montevideo. Revista Uruguaya de Enfermería, 1, 25-39.

Monroy, B. \& Palacios, L. (2011). Resiliencia: iEs posible medirla e influir en ella? Salud Mental, 34(3), 237-246.

Munist, M., Santos, H., Kotliarenco, M. A., Suárez, E., Infante, F. \& Grotberg, E. (1998). Manual de identificación y promoción de la resiliencia en niños y adolescentes. Washington DC: Organización Panamericana de la Salud.
Omar, A. (2009). Las perspectivas de futuro y sus vinculaciones con el bienestar y la resiliencia en adolescentes. Revista Psicodebate: Psicología, Cultura y Sociedad, 7, 141-154.

Palomar, J. \& Gómez, N. (2010). Desarrollo de una escala de medición de la resiliencia con mexicanos. Interdisciplinaria, 27(1), 7-22. Recuperado de http:// www.redalyc.org/articulo.oa?id=18014748002

Rutter, M. (1979). Protective factors in children's responses to stress and disadvantages. En M. W. Kent \& J. E. Rolf (Eds.), Primary prevention of psycopathology. Social competence in children (Vol. 3, pp. 49-74). Hanover, NH: University Press of New England.

Rutter, M. (1993). Resilience: Some conceptual considerations. Journal of adolescent health, 14(8), 626-631. http://dx.doi.org/10.1016/1054139X(93)90196-V

Salgado, A. C. (2005). Métodos para medir la resiliencia: una alternativa peruana. Liberalit. Revista de Psicología, 11, 41-48.

Suárez, E. N. (1996). El concepto de resiliencia desde la perspectiva de la promoción de salud. En M. A. Kotliarenco, C. Álvarez \& I. Cáceres (Comps.), Resiliencia: construyendo en adversidad (pp. 51-64). Santiago, Chile: CEANIM. Recuperado de http:// www.resiliencia.cl/investig/Res-CAdversidad.pdf

Vanistendael, S. (1997, junio). Resiliencia. Conferencia presentada en el Seminario Los Aportes del Concepto Resiliencia en los Programas de Intervención Psicosocial, Santiago, Chile.

Vera, B., Carbelo, B. \& Vecina, M. L. (2006). La experiencia traumática desde la psicología positiva: resiliencia y crecimiento postraumático. Papeles del Psicólogo, 27(1), 40-49.

Wagnild, G. M. \& Young, H. M. (1993). Development and psychometric evaluation of the Resiliency Scale. Journal of Nursing Measurement, 1(2), 165 178.

Windle, G., Bennett, K. M. \& Noyes, J. (2011). Methodological review of resilience measurement scales. Health and Quality of Life Outcomes, 9(8), 1-18.

Windle, G., Markland, D. A. \& Woods, B. (2008). Examination of a theoretical model of psychological resilience in older age. Aging $8 \mathcal{8}$ Mental Health, 12(3), 285-292. 